\title{
Accuracy of Ultrasound Compared to MRI in Evaluation of Rotator Cuff Tears
}

\author{
Poorvi Malikㅜ, Kamal Kumar Sen ${ }^{2}$, Manoj Kumar ${ }^{3}{ }^{3}$, Nilanjan Mukherjee ${ }^{4}$, Manoj Kumar Veerabathini ${ }^{5}$, Aavula \\ Adarsh $^{6}$

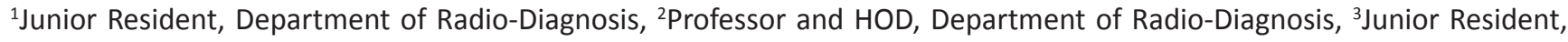 \\ Department of Radio-Diagnosis, 4 Junior Resident, Department of Radio-Diagnosis, 5Junior Resident, Department of Radio-

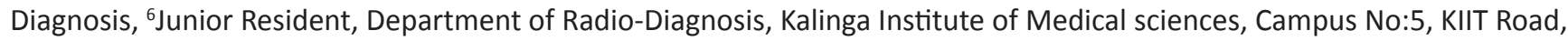 \\ Patia, Bhubaneswar, Odisha 751024, India
}

Corresponding author: Dr. Kamal Kumar Sen, Professor, Department of Radio-Diagnosis, Kalinga Institute of Medical Sciences, Campus No:5, KIIT Road, Patia, Bhubaneswar, Odisha 751024, India

DOI: http://dx.doi.org/10.21276/ijcmsr.2020.5.1.5

BY-NC-ND

How to cite this article: Poorvi Malik, Kamal Kumar Sen, Manoj Kumar G, Nilanjan Mukherjee, Manoj Kumar Veerabathini, Aavula Adarsh. Accuracy of ultrasound compared to MRI in evaluation of rotator cuff tears. International Journal of Contemporary Medicine Surgery and Radiology. 2020;5(1):A21-A25.

\section{A B S T R A C T}

Introduction: Rotator cuff pathologies consist of a significant proportion of shoulder pathologies. Multiple imaging modalities are available for evaluation of shoulder. However, ultrasound and Magnetic Resonance Imaging (MRI) are the most commonly used. MRI has the advantage of providing excellent soft tissue resolution and multi-planar imaging. Ultrasound however, is much cheaper, but has a long learning curve. Aim of the study is to evaluate the accuracy of Ultrasound findings in relation to Magnetic Resonance Imaging in the patients with shoulder pain for pretreatment evaluation.

Material and Methods:100 patients of suspected shoulder pathology were studied. Sonography was performed using high frequency linear transducer 6-12MHz in GE Voluson S6 Scanner, followed by Magnetic Resonance Imaging with GE Signa HDxT1.5 Tesla MRI using surface coil.

Results: The most common rotator cuff tendon involved was supraspinatus. On comparison with Magnetic Resonance Imaging, sonographic detection of tendinosis had a sensitivity of $77.27 \%$ and specificity of $89.45 \%$. Partial thickness tears showed a sensitivity of $59.68 \%$ and specificity of $99 \%$ and full thickness tears showed a sensitivity of $100 \%$ and specificity of $99.63 \%$. Also comparable accuracy amongst the two modalities was seen for evaluation of long head of biceps tendon pathologies, retraction of muscle fibers and fatty atrophy of supraspinatus muscle. Conclusion: Pretreatment evaluation of suspected rotator cuff pathologies could include Ultrasound as first line imaging modality. However, MRI should be used as a complementary modality for further assessment of cause or identifying source of symptoms in patient presenting with shoulder pain.

Keywords: Ultrasound, Magnetic Resonance Imaging, Rotator Cuff Tear, Shoulder Pain

\section{INTRODUCTION}

Shoulder pain with or without trauma is a common complaint in day to day life. The shoulder is anatomically complex with numerous structures contributing to both the mobility and stability of the joint. Variations of normal anatomy can lead to confusion during arthroscopic diagnosis and treatment ${ }^{1}$ Pathologically the three most important entities affecting the shoulder are the rotator cuff pathologies, impingement, and instability. Commonly used imaging modalities are plain radiograph, Ultrasound (US), Computed tomography (CT) and Magnetic Resonance Imaging (MRI). Plain radiographs form the first line of investigation but soft tissue injuries are missed. Disadvantages of CT include ionizing radiation and poor soft tissue delineation.

Ultrasound has its advantage being dynamic examination, easy availability, quick scan time, and often allows therapeutic interventions with better patient compliance., ${ }^{2,3}$ MRI provides excellent soft tissue delineation and evaluation of both intra-articular and extra-articular anatomy. ${ }^{1}$ Moreover MRI is the modality that can assess the composition of bone marrow or edema related to trauma and other conditions. ${ }^{4}$ In this study we compare the role of high resolution Ultrasonography with MRI for various rotator cuff pathologies presenting with shoulder pain.

\section{MATERIAL AND METHODS}

An observational, prospective study at our tertiary care hospital, in consecutive 100 patients presenting with shoulder trauma/pain and suspected of having rotator cuff pathology was done after clearance from institutional ethical committee.

Patients who had undergone either MRI or only US, postoperative patients and patients with associated referred pain were excluded from the study. First US was performed in each and then followed by MRI 
Ultrasonography was done using high frequency linear transducer 6-12MHz with GE Voluson S6. The examination was done in sitting position. The standard protocol was followed for systematic examination of all rotator cuff tendons and long head of biceps tendon using various dynamic maneuvers. Modified crass position was used for supraspinatus evaluation. Also subacromial subdeltoid bursa and acromioclavicular joint were examined simultaneously.

MRI was done in all patients on GE Signa HDxT 1.5 Tesla MRI using surface coil. Magnetic resonance imaging studies was performed in three orthogonal planes for all patients. The coronal oblique sequence was oriented parallel to the long axis of the body of the scapula and the supraspinatus tendon (not the muscle) and perpendicular to the tangent line passing from glenoid fossa. The routine coronal, axial and sagittal T2 w and Proton Density Fat Saturated sequence was taken. Axial Gradient Echo and Sagittal T1 W sequences were also performed whenever indicated. Slice thickness was 3-5mm (average 4mm) and FOV: 16 - $20 \mathrm{~cm}$ with Matrix Size: $256 * 224$.

For pretreatment evaluation the following were reported in US and MRI on standard format-

Muscle(s) involved [supraspinatus (SS), Subscapularis
(SCP), Infraspinatus (IFS), Long Head of Biceps Tendon (LHBT)]

Tendinosis/Partial thickness (PT) /Full thickness (FT)tear Surface of the tendon involved in tear (articular; bursal; intrasubstance)

Extent of retraction of the torn muscle

Atrophy of the muscle involved

Associated bony changes, joint fluid and peribicipital fluid collection, subacromial-subdeltoid fluid collection, labral pathologies and impingement.

\section{STATISTICAL ANALYSIS}

Descriptive statistics for clinical parameters were reported as frequency [in \%] for qualitative variable \& mean+/- SD for quantitative parameter. The diagnostic measures such as sensitivity, specificity, positive predictive value, negative predictive value and overall diagnostic accuracy was calculated. All the analysis was carried out with the help of standard statistical analysis methods.

\section{RESULTS}

Demographic Data: In our study most of the patients belonged to the $>40-60$ years age group. Mean age was 45.2

\begin{tabular}{|c|c|c|c|c|c|c|c|}
\hline S. No & Muscle & Findings & $\begin{array}{c}\text { Sensitivity } \\
\text { (\%) }\end{array}$ & $\begin{array}{c}\text { Specificity } \\
\text { (\%) }\end{array}$ & PPV (\%) & NPV (\%) & Accuracy (\%) \\
\hline \multirow[t]{3}{*}{1} & \multirow[t]{3}{*}{ Supraspinatus } & Tendinosis & 88.46 & 82.43 & 63.89 & 95.31 & 84 \\
\hline & & Partial Thickness tear & 60.00 & 98.57 & 94.74 & 85.19 & 87 \\
\hline & & Full Thickness tear & 100.0 & 100.0 & 100.0 & 100.0 & 100.0 \\
\hline \multirow[t]{3}{*}{2} & \multirow[t]{3}{*}{ Subscapularis } & Tendinosis & 64.17 & 87.95 & 52.38 & 92.41 & 84.00 \\
\hline & & Partial Thickness tear & 54.17 & 98.68 & 92.86 & 87.21 & 88.00 \\
\hline & & Full Thickness tear & 100.00 & 98.95 & 83.33 & 100.00 & 99.00 \\
\hline \multirow[t]{3}{*}{3} & \multirow[t]{3}{*}{ Infraspinatus } & Tendinosis & NC & 95.96 & NC & 98.96 & 95.00 \\
\hline & & Partial Thickness tear & 75.00 & 100.00 & 100.00 & 97.87 & 98.00 \\
\hline & & Full Thickness & 100.00 & 100.00 & 100.00 & 100.00 & 100.00 \\
\hline \multirow[t]{3}{*}{4} & \multirow[t]{3}{*}{ LHBT } & Tendinosis & 73.68 & 98.77 & 93.33 & 94.12 & 94.00 \\
\hline & & Partial Thickness tear & 87.50 & 100.00 & 100.00 & 98.62 & 99.00 \\
\hline & & Full Thickness & 100.00 & 100.00 & 100.00 & 100.00 & 100.00 \\
\hline
\end{tabular}

\begin{tabular}{|l|l|c|c|c|}
\hline MRI findings & Positive & Negative & Total \\
\hline USG & Positive & 23 & 0 & 23 \\
\hline & Negative & 3 & 74 & 77 \\
\hline & Total & 26 & 74 & \\
\hline Sensitivity (\%) & Specificity (\%) & PPV (\%) & NPV (\%) & Diagnostic Accuracy (\%) \\
\hline 88.46 & 100 & 100 & 96.1 & 97 \\
\hline \multicolumn{2}{|l}{} \\
\hline
\end{tabular}

\begin{tabular}{|l|l|c|c|c|}
\hline MRI Findings & Positive & Negative & Total \\
\hline USG & Positive & 13 & 0 & 13 \\
\hline & Negative & 4 & 83 & 87 \\
\hline & Total & 17 & 83 & \\
\hline Sensitivity (\%) & & & & \\
\hline 74.47 & Specificity (\%) & PPV (\%) & NPV (\%) & Diagnostic Accuracy (\%) \\
\hline \multicolumn{2}{|l|}{100} & 95.4 & 96 \\
\hline
\end{tabular}




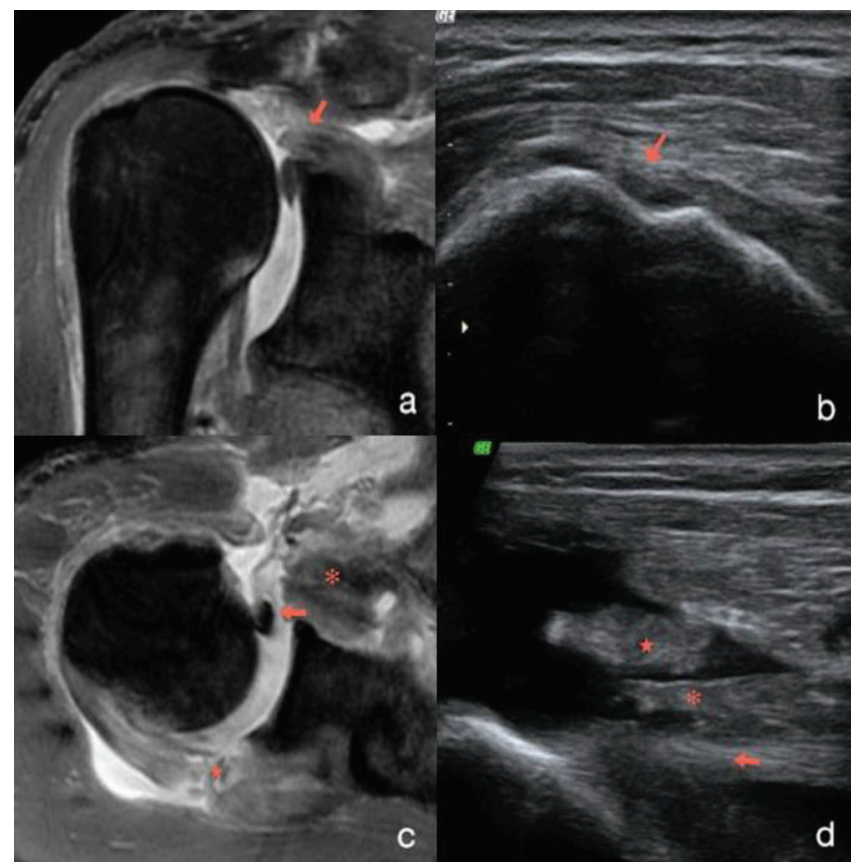

Figure-1: MRI Coronal PDFS shows superior translation of humeral head $(\mathrm{HH})$ with complete full thickness tear of supraspinatus (arrow)with retraction of fibers beyond glenoid labrum (a). Moderate joint effusion also seen. Transverse USG scan shows non visualisations of the supraspinatus fibers (arrow), suggestive of full thickness tear (b). MRI Axial PDFS shows full thickness tear of subscapularis tendon (asterisk) with retraction of fibers upto the glenoid. Also note the full thickness tear of infraspinatus muscle (star). Biceps tendon is dislocated (arrow) from the groove with hyper intensity within suggestive of tendinosis(c). Transverse scan shows retraction of supraspinatus (star) and subscapularis fibers (asterisk) with moderate joint effusion. The deeper muscle fibers correspond to the biceps tendon (arrow) which appears taut and intact (d).

- Supraspinatus pathology Bursitis

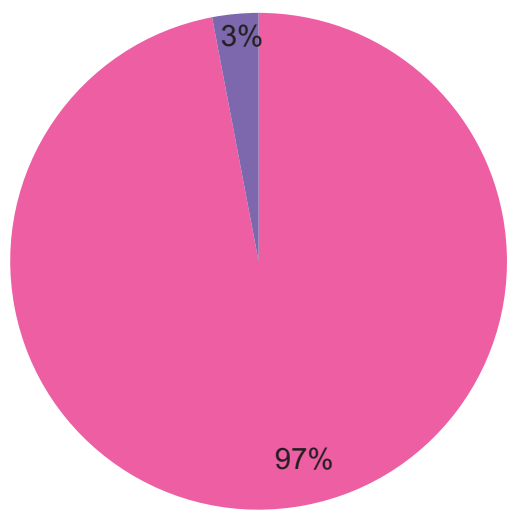

Graph-1: Associated pathologies with subacromial subdeltoid bursa fliuid collection

years and SD 15.5 years. A clear male gender predilection, with a male to female ratio of $4.5: 1$ was observed in this study.

Clinical Presentation: Amongst patients aged $>40 y e a r s$, $62.2 \%$ of patients had traumatic rotator cuff tear rest had degenerative rotator cuff tear. About 26\% patients had restriction of movements amongst the study population. This posed a diagnostic challenge during performance of dynamic maneuvers in ultrasound.

The most common rotator cuff tendon involved was supraspinatus and the most common pathology associated being partial thickness tears. All the 22 FT tears detected on MRI were confidently diagnosed on US. However, approximately half of partial thickness tears were either missed or misdiagnosed as tendinosis on US. Only one case reported as normal on MRI was misdiagnosed as tendinosis on US. (Figure 1)

Most common pathology in Subscapularis was partial thickness tears. Amongst 5 FT tears, all were correctly identified on US also. However, 5 tendinosis were not identified on ultrasound and 1 was misdiagnosed as PT tear. 10 PT tears were misdiagnosed as tendinosis and 1 was missed on US. (Figure 1)

Most common pathology we found in infraspinatus was Partial thickness tears. Amongst 4 Full Thickness tears of Subscapularis, all were correctly identified on US also. Two Partial Thickness tears were misdiagnosed, one as Tendinosis and 1 was missed on USG. (Figure 1)

The diagnostic accuracy of ultrasound in detection of SS tendinosis, partial and full thickness tears is $84 \%, 87 \%$ and $100 \%$ respectively. Full thickness tears had 100\% sensitivity, specificity, NP V, PPV, diagnostic accuracy. For ultrasound detection of partial thickness tears we found the specificity (98.57\%) to be more than sensitivity (60\%), whereas for tendinosis higher sensitivity (88.46\%) was found. (Table 1 )

The diagnostic accuracy of ultrasound in detection of SCP tendinosis, partial and full thickness tears is $84 \%, 88 \%$ and 99\% respectively. Full thickness tears had 100\% sensitivity, 98.95\% specificity, 100\% NPV, 83.33\% PPV, 99\% diagnostic accuracy. For ultrasound detection of partial thickness tears, we found the specificity (98.68\%) to be more than sensitivity (54.17\%), whereas for tendinosis also we had a higher specificity (87.95\%) than sensitivity (64.71\%). (Table 1)

The diagnostic accuracy of US in detection of IFS tendinosis, PT and FT is $95 \%, 98 \%$ and $100 \%$ respectively. FT tears had 100\% sensitivity, specificity, NPV, PPV, diagnostic accuracy. For US detection of PT tears, we found the specificity (100\%) to be more than sensitivity (75\%), similarly for tendinosis higher sensitivity (95.96\%) was found. (Table 1)

Only 4 cases each of subluxation and dislocation of LHBT were identified in our study and all were correctly diagnosed, providing $100 \%$ accuracy. However, for LHBT tendinopathy 14 cases were correctly identified on ultrasound and 5 were misdiagnosed giving a total accuracy of $94 \%$. Only 1 case of FT tear of LHBT was seen and correctly identified on US. For PT tears an accuracy of $99 \%$ was found in our study. (Table 1) (Figure 1)

The combined accuracy for diagnosis of tendinosis of any rotator cuff muscle is $87.67 \%$, while for partial thickness tears it is $91 \%$ and full thickness tears it is $99.67 \%$. The specificity for all the afore mentioned pathologies is more than the sensitivity in our study.

In our study $97 \%$ of subacromial subdeltoid bursal fluid collections were associated with supraspinatus pathologies (predominantly tears) and only 3\% of isolated subacromial 
subdeltoid bursitis was found. (Graph1)

Most cases of biceps effusion (79\%) were associated with rotator cuff tears in our study.

A total of 26 patients with SS tear had retraction of muscle fibers. Amongst these 23 were correctly identified in our study. Ultrasound had a near equal accuracy (97\%) as compared to MRI for diagnosis of retraction of SS fibers in rotator cuff tears especially beyond glenoid. (Table 2)

A total of 17 patients with SS tear had fatty atrophy of supraspinatus muscle. Amongst these 13 were correctly identified in our study. Thereby, USG provided a near equal diagnostic accuracy of $96 \%$ for diagnosis of fatty atrophy in patients with rotator cuff tears. (Table 3 )

Ultrasound showed good detection rate of bony changes as MRI, especially avulsion injuries associated with greater tuberosity fractures.

\section{DISCUSSION}

About $60 \%$ of patients with $>40 \mathrm{yrs}$ of age and rotator cuff tear had history of trauma. Rest $40 \%$ had a degenerative tear correlating with the previous studies by Neer et al describing most of tears in old patients as degenerative tears. ${ }^{5}$ About 26 patients amongst 100 study population had restriction of movements. This led to decreased efficacy of ultrasound in our study, especially during the dynamic maneuvers.

In our study the most common rotator cuff tendon involved in pathology was supraspinatus tendon (78\%) followed by subscapularis tendon and infraspinatus tendon. Most common supraspinatus pathology found was partial thickness tears amongst which articular surface tears were the more common ones. For supraspinatus muscle on comparison to MRI we found that ultrasound could detect all the full thickness tears with $100 \%$ accuracy, sensitivity and specificity. We speculate that this was mainly because most of the full thickness tears had moderate effusion providing us good acoustic window for visualization of muscle pathologies.

Most of these tears were associated with retraction of fibers giving us a diagnostic clue. Amongst 30partial thickness tears of supraspinatus, 16 were detected on ultrasound, 13 were misdiagnosed as tendinosis and 1 low grade tear was not detected. This could be due to chronic nature of tears due to which they appear more heteroechoic than anechoic leading to an inaccurate interpretation. Chronic tears are also associated with minimal to no effusion providing poor window for visualization on Ultrasound.

Partial thickness tears of higher grade and the ones with associated signs (most commonly subacromial subdeltoid bursal fluid collection, bony irregularities or associated fractures) could be detected on Ultrasound. Overall we found $94 \%$ accuracy for detection of partial thickness supraspinatus tears.

For subscapularis tears we found 5 full thickness tears with $100 \%$ sensitivity, $98.5 \%$ specificity and $99 \%$ accuracy of detection on US. For 24 partial thickness tears of subscapularis, on Ultrasound 13 were correctly diagnosed. This could be due to the limitation of external rotation in these patients usually associated with subscapularis tears. Most of these subscapularis tears were located near musculotendinous junction (where the muscle fibers go deep to the coracoid process) in our study and the visualization of the same on ultrasound is usually inaccurate due to anisotropy on transverse scan.

For infraspinatus tendon most common pathology was partial thickness tears, all of which were articular surface tears. We found a good accuracy rate of $94 \%$ for detection of these tears. Four full thickness tears were found on MRI and all were detected accurately on Ultrasound.

All over for detection of rotator cuff pathologies on US we found tendinosis to have a sensitivity of $77.27 \%$ and specificity of $89.45 \%$. Partial thickness tears showed a sensitivity of $59.68 \%$ and specificity of $99 \%$ and for full thickness tears we found a sensitivity of $100 \%$ and specificity of $99.63 \%$.

These results were similar to the metaanalysis of Jesus et al. They found sensitivity of $66.7 \%$ and specificity of $93.5 \%$ in partial thickness and $92.3 \%$ sensitivity and $94.4 \%$ specificity in full thickness tears. ${ }^{6}$

Similar results were seen in the meta analysis of Roy et al who found $91 \%$ sensitivity, $93 \%$ specificity for full thickness tears. $68 \%$ sensitivity, $94 \%$ specificity for partial thickness tears and $79 \%$ sensitivity and $94 \%$ specificity for tendinosis. ${ }^{7}$ However, in our study we found $100 \%$ sensitivity and 99.63\% specificity and diagnostic accuracy of $99.67 \%$ for full thickness tears similar to the study by Anastasia et $\mathrm{al}^{8}$ who found an accuracy of $98 \%$ for full thickness tears. This discrepancy could be due to the selection bias since most cases had positive findings in our study and presented with trauma and extensive joint effusion.

For long head of biceps tendon pathology Skendzel et $\mathrm{al}^{9}$ described an accuracy of $88 \%$ and $97 \%$ for detection of partial and full thickness tears respectively.

In our study we found a diagnostic accuracy of $99 \%$ and $100 \%$ for partial and full thickness tears. We speculate the discrepancies of aforementioned findings could be due to decreased sample size in our study (no of cases of partial thickness (four) and full thickness tear (one)).

For pretreatment evaluation of patient and prognostic implication, orthopedic surgeons also require particulars about atrophy and fatty degeneration of ruptured muscle, retraction of fibers in tears and associated bony changes. Retraction of supraspinatus fibers beyond glenoid carry bad prognosis..$^{10}$ For the assessment of retraction of fibers in supraspinatus tears we found $88.4 \%$ sensitivity and $100 \%$ specificity similar to the findings of Alagappan et al. ${ }^{11}$

Fatty Degeneration and atrophy of muscle correlates with the level muscle function that could be revived following surgery, helping in deciding surgical treatment or medical management for many patients. ${ }^{10}$ Fatty degeneration evaluation was done for supraspinatus muscle only in our study by Goutallier staging. ${ }^{12}$ We found a sensitivity of USG of $74.4 \%$ and diagnostic accuracy of $96 \%$. These findings correlate with Lindley B. Wall et al who found a similar sensitivity of $84.6 \% .^{13}$

Limitations: MR arthrography was not performed in any of the cases, which could have helped us delineate the very small partial thickness tears. Inter observer variability was not been assessed in our study since all images were evaluated by a single radiologist. 


\section{CONCLUSION}

Other than being a cost effective, readily available modality USG has an added advantage of providing superior resolution by high frequency transducers. It also has the ability to perform real time evaluation and dynamic maneuvers, especially to confirm cases of subacromial or sub coracoid impingement and subluxation of biceps. Another benefit was convenient comparison with the contralateral normal shoulder which is generally not undertaken in MR Imaging since it will not be cost effective.

In our study we also evaluated accuracy of ultrasound compared to MRI for pretreatment and prognostic information required (retraction of muscle fibers in supraspinatus tears up to glenoid bone and fatty atrophy of supraspinatus muscle) in a rotator cuff tear. Comparable accuracy was found between both modalities.

Other than being used in rotator cuff and long head of biceps tendon pathology, role of US in assessment of bony abnormalities (e.g. Acromioclavicular joint arthropathy, greater tuberosity fractures, cortical irregularities at supraspinatus footprint and large hill sacks lesions) were well evaluated with good accuracy in our study.

Ultrasound however has certain limitations, most important being operator dependence, a long learning curve, artefacts like anisotropy and difficulty in diagnosis of chronic tears. Also the cause for tears in patients other than acute traumatic presentation could not always be assessed for which further MRI Imaging is advisable.

Role of ultrasound in bursal fluid collection and joint effusion is already well established. However, we also found that very small subacromial collection could not be detected in ultrasound due to posterior acoustic shadowing from acromion process. This being an indicator of degenerative bursal surface tear, especially in elderly age group, MR imaging is suggested in such cases.

In the end we conclude that ultrasound could safely be considered as first line imaging modality in patients presenting with shoulder pain with suspected rotator cuff pathology for complete pretreatment evaluation. However, MRI should be used as a complementary modality for assessment of cause or identifying source of symptoms in patient presenting with shoulder pain.

\section{REFERENCES}

1. Phillip F. J. Tirman, Lynne S. Steinbach, John P. Belzer, and Frederic W.Bost, A Practical Approach to imaging of shoulder with emphasis on MRImaging. Orthopaedics Clinics of North America.1997.

2. Gupta A, Gupta H. The radiological assessment of shoulder pathology,Orthopaedics and Trauma (2018)

3. Steve Gazzolaand Robert R. Bleakney.Current Imaging of the Rotator Cuff.Sept 2011;

4. Pranshu Sharma, William B. Morrison, Steven Cohen. Imaging of the Shoulderwith Arthroscopic Correlation. Clin Sports Med . 2013

5. David W. Stoller. Magnetic Resonance Imaging in Orthopaedics and sportsMedicine. 3rd edition. p11311462

6. Joseph O. de Jesus, Laurence Parker Andrea J.
FrangosLevon N. Nazarian. Accuracy of MRI, MR Arthrography, and Ultrasound in the Diagnosis ofRotator Cuff Tears: A Meta-Analysis. American Journal of Roentology .2009

7. Jean-Sébastien Roy, Caroline Braën, Jean Leblond, FrançoisDesmeules, Clermont E Dionne, Joy C MacDermid, Nathalie J Bureau, PierreFrémont . Diagnostic accuracy of ultrasonography, MRI and MR arthrographyin the characterisation of rotator cuff disorders: a meta-analysis . Br J SportsMed 2015

8. A.N. Fotiadou, Marianna Vlychou, Periklis Papadopoulos, Dimitrios S.Karataglis, Panagiotis Palladas, Ioannis V. Fezoulidis. Ultrasonography ofsymptomatic rotator cuff tears compared with MR imaging and surgery. European Journal of Radiology. 2008

9. Jack G. Skendzel, Jon A. Jacobson, James E. Carpenter, Bruce S. Miller. Long Head of Biceps Brachii Tendon Evaluation: Accuracy of PreoperativeUltrasound. American Journal of Roentology.2011

10. Morag Y1, Jacobson JA, Miller B, De Maeseneer M, Girish G, Jamadar D. MR imaging of rotator cuff injury: what the clinician needs to know. Radiographics.2006

11. Vinod Alagappan et al. Imaging of rotator cuff pathology: is ultrasound as goodas MRI.Journal of Evolution of Medical and Dental Sciences .2015

12. Khoury V, Cardinal É, Brassard P. Atrophy and fatty infiltration of the supraspinatus muscle: sonography versus MRI. American Journal of Roentgenology. 2008.

13. Lindley B. Wall, Sharlene A. Teefey, William D. Middleton, NirvikarDahiya, MD, Karen StegerMay, MA, H. Mike Kim, MD, Daniel Wessell, KenYamaguchi. Diagnostic Performance and Reliability of Ultrasonography forFatty Degeneration of the Rotator Cuff Muscles. Journal Bone Joint Surg Am.2012

Source of Support: Nil; Conflict of Interest: None

Submitted: 27-11-2019; Accepted: 31-12-2019; Published online: 17-01-2020 